\title{
Profile of Bacteria with ARGs Among Real-World Samples from ICU Admission Patients with Pulmonary Infection Revealed by Metagenomic NGS
}

\author{
Huijuan Chen' \\ Xinhua $\mathrm{Bai}^{2}$ \\ Yang $\mathrm{Gao}^{2}$ \\ Wenxuan $\mathrm{Liu}^{2}$ \\ Xuena $\mathrm{Yao}^{2}$ \\ Jing Wang ${ }^{2}$
}

'Department of Biomedical Engineering, College of Life Science and

Bioengineering, Beijing University of

Technology, Beijing, People's Republic of

China; ${ }^{2}$ Department of Clinical

Laboratory, Beijing Capitalbio Medlab,

Beijing, People's Republic of China
Correspondence: Huijuan Chen

Tel +86-10-8722-9999

Email m13810539097@I63.com
Background: Treatment of pulmonary infections in the intensive care unit (ICU) represents a great challenge, especially infections caused by antibiotic resistance pathogens. A thorough and up-to-date knowledge of the local spectrum of antibiotic resistant bacteria can improve the antibiotic treatment efficiency. In this study, we aimed to reveal the profile of bacteria with antibiotic resistance genes (ARGs) in real-world samples from ICU admission patients with pulmonary infection in Mainland, China, by metagenomic next-generation sequencing (mNGS).

Methods: A total of 504 different types of clinical samples from 452 ICU admission patients with pulmonary infection were detected by mNGS analysis.

Results: A total of 485 samples from 434 patients got successful mNGS results. Among 434 patients, one or more bacteria with ARGs were detected in 192 patients $(44.24 \%, 192 / 434)$, and $\geq 2$ bacteria with ARGs were detected in $85(19.59 \%, 85 / 434)$ patients. The predominant detected bacteria were Corynebacterium striatum (C. striatum) (11.76\%, 51/434), Acinetobacter baumannii (A. baumannii) (11.52\%, 50/434) and Enterococcus faecium (E. faecium) $(8.99 \%, 39 / 434)$. erm $X$ conferred resistance to $\mathrm{MSL}_{\mathrm{B}}$ and $c m x$ to phenicol were the only two ARGs detected in C. striatum; in A. baumannii, most of ARGs were resistance-nodulation-division (RND)-type efflux pumps genes, which conferred resistance to multi-drug; ermB conferred resistance to $\mathrm{MSL}_{\mathrm{B}}$ and efmA to multi-drug were the predominant ARGs in E. faecium. Bacteria with ARGs were detected in 50\% (140/280) bronchoalveolar lavage fluid (BALF) and 50.5\% (48/95) sputum samples, which were significantly higher than in blood and cerebrospinal fluid (CSF) samples.

Conclusion: High level of bacteria with ARGs was observed in clinical samples, especially BALF and sputum samples from ICU admission patients with pulmonary infection in Mainland, China. And C. striatum resistant to $\mathrm{MSL}_{\mathrm{B}}$ and/or phenicol, multi-drug resistance $A$. baumannii and E. faecium were the lead bacteria.

Keywords: intensive care units, pulmonary infection, antibiotic resistant bacteria, antibiotic resistance genes

\section{Introduction}

Pulmonary infection is one of the most frequent nosocomial infections in the intensive care unit (ICU) admission patients and is associated with significant morbidity and mortality. Patients developing pneumonia while in the ICU have a high crude mortality rate of approximately $30 \%{ }^{1}$ Currently, the empirical choice of broad-spectrum antibiotic therapy is standard-of-care therapy for most patients with infection in the ICU, 
especially in critically ill patients. Clinical success rates reported for patients who developed pneumonia in the ICU remain low (often $<60 \%$ ), ${ }^{2}$ relapse rates are high, despite the widespread use of broad-spectrum antibiotics. Mortality rate is significantly lower in patients who received appropriate antibiotic therapy at the early stage of nosocomial pneumonia compared with the patients who did not receive or delayed appropriate therapy. ${ }^{7}$ An exponential increase of antibiotic drug resistant microbes in ICU, especially the multi-drug resistance (MDR) pathogens, is the biggest challenge that encompass antibiotic therapy. An international multi-center study demonstrated that $19 \%$ of patients with ICU stays over 24 hours acquired an infection, ranging from $2.3 \%$ to $49.2 \%$ depending on the hospital unit. ${ }^{8}$ The average for infections by multi-resistant gram-negative bacteria in ICU patients was 54.4 per 1000 patients/day. ${ }^{9}$ MDR pathogens, Enterococcus faecium (E. faecium), Staphylococcus aureus (S. aureus), Klebsiella pneumonia (K. pneumonia), Pseudomonas aeruginosa (P. aeruginosa), Acinetobacter baumannii (A. baumannii), Enterobacter species have been classified as ESKAPE $^{3}$ and are the most common microbial causes of ICU acquired infection including the pulmonary infection, ${ }^{4}$ but causative pathogens and antibiotics resistance patterns are highly endemic. A thorough and up-to-date knowledge of the local microbial cause epidemiology might allow better initial empirical antibiotic choice to susceptible microorganisms in ICU, while avoiding the overuse of broad-spectrum antibiotic and the emergence of new drugresistant organism. ${ }^{5,6}$ Many genes contributing to antibiotic resistance have been identified, and the high concordance of genotype-phenotype for drug resistance have been reflected along with the broader usage of NGS technologies in evaluating the antimicrobial resistance (AMR) genetic determinants. ${ }^{61,62}$ Metagenomic next-generation sequencing (mNGS) has the capacity to characterize all DNA or RNA present in a sample, enabling analysis of all potential pathogens and characterization of antibiotic resistance directly from clinical samples. To date, several studies have demonstrated the utility of mNGS in providing clinically actionable information to predict cause of infection and evaluate disease risk, and the effectiveness of mNGS in guiding patient management. ${ }^{53-57}$ In this study, we detected different types of clinical samples from ICU admission patients with pulmonary infection using a validated mNGS assay, in order to reveal the prevalence and distribution of bacteria with antibiotic resistance genes (ARGs) in samples of real-world ICU admission patients with pulmonary infection in Mainland, China.

\section{Materials and Methods}

\section{Study Population and Specimens}

This retrospective study included 452 ICU admission patients with pulmonary infection who underwent clinical metagenomics next-generation sequencing (mNGS) testing in CapitalBio Medlab (Beijing, China) from January 2019 to November 2020. All patients were diagnosed as pulmonary infection judged by the board-certified physician on the basis of clinical manifestations and imaging examination results. ${ }^{59,60} \mathrm{mNGS}$ analysis was ordered as clinically indicated by the treating physician of patients, and informed consent was obtained from each included patient. Total 504 clinical specimens from the 452 patients were included, including Bronchoalveolar lavage fluid (BALF, $\mathrm{n}=284)$, sputum $(\mathrm{n}=97)$, peripheral blood $(\mathrm{PB}, \mathrm{n}=97)$, cerebrospinal fluid (CSF, $\mathrm{n}=14)$, pus $(\mathrm{n}=3)$, pleural fluid $(\mathrm{PF}, \mathrm{n}=5)$ and lung biopsy tissue $(\mathrm{n}=4)$. Two different types of specimen were collected from 52 patients simultaneously.

\section{Sample Collection and Processing}

All body fluid samples (BALF, sputum, pleural fluid) were collected from each patient according to standard procedures. ${ }^{63}$ At least $5 \mathrm{~mL}$ of BALF sample and $1-3 \mathrm{~mL}$ of each sample of sputum, CSF, pleural fluid or other sterile liquid were collected in sterile tube and stored at $-80^{\circ} \mathrm{C}$ before DNA extraction. Sputum samples were liquefied by dithiothreitol (DTT) for $30 \mathrm{~min}$ at room temperature with shaking, centrifuged and washed by sterile water twice before DNA extraction. Other body fluid samples except blood could go directly to the DNA extraction procedures. $3-5 \mathrm{~mL}$ peripheral blood was collected to Ethylene Diamine Tetraacetic Acid (EDTA) anticoagulant tube with special deoxyribonucleic acid (DNA) protective agent and stored at room temperature. The blood samples were centrifuged at $1600 \mathrm{~g}$ for $10 \mathrm{~min}$ to separate plasma, and the plasma separation must carried out within 48 hours after blood draw. Lung biopsy tissue was minced into small pieces and ground with a tissue homogenate machine before DNA extraction. $0.6 \mathrm{~mL}$ sample, $20 \mu \mathrm{L}$ enzyme, and $1 \mathrm{~g}$ of $0.5 \mathrm{~mm}$ Zirconium beads in a new, sterile $1.5 \mathrm{~mL}$ microcentrifuge tube were shaken vigorously by a vortex mixer and $0.5-\mathrm{mL}$ sample was transferred to a new $1.5 \mathrm{~mL}$ microcentrifuge tube for DNA extraction. DNA was extracted using the MAPMI sample preparation kit (CapitalBio Corporation, Beijing, China) following the manufacturer's operational manual. 


\section{Library Preparation and Sequencing}

DNA libraries were constructed through enzymatically DNA fragmentation (200-300bp), end repair, adapters adding, and PCR amplification. The quality of the DNA libraries was assessed using an Agilent 2100 Bioanalyzer (Agilent Technologies, Santa Clara, California) combined with qPCR based on Applied Biosystems 7500 Real-Time PCR System (Thermo Fisher, USA), the primers based on the sequences of the adapters were used for the qPCR. Libraries were out of the range of 300-400bp, or with a concentration $<50 \mathrm{pmol} / \mathrm{L}$, considered to have failed the library preparation step. Constructed libraries with confirmed quality were pooled and sequenced with Ion PI chip on BES4000 (Capitalbio Corporation, Beijing, China) platform. Internal, negative and positive controls were included in each run. Internal parameters are specific molecular tag that is placed in the sample before nucleic acid extraction to track the entire process and to control the quality of DNA. Negative control is sterile water to monitor potential contamination and positive control is a mock community comprising genomic DNA prepared from 8 bacteria/ fungi species mixed at varying concentrations.

\section{Bioinformatic Analysis}

An automated bioinformatic analysis system (Capitalbio Corporation, Beijing, China) based on Ion torrent ${ }^{\mathrm{TM}}$ server combined with BES4000 sequencer was used for data analysis. 1) Quality control: The original sequencing data were analyzed if: (i) Sequencing sequences subtracted of human host sequences was above $70 \%$. (ii) Reads of sequence were longer than $100 \mathrm{bp}$. (iii) Total reads without removing the human genome component were $>10$ million. 2) Data filtering: in order to obtain highquality sequence data, the reads with length less than $50 \mathrm{bp}$, low-quality, low complexity were removed. Host subtraction was performed by mapping to human reference genome (GRCh38). 3) sequences alignment: The remaining un-mapped reads were aligned directly with large reference databases downloaded from NCBI, PATRIC and Comprehensive Antibiotic Research Database (CARD) databases, which cover bacteria, fungi, viruses, parasite and ARGs. According to the final results of microorganisms and ARGs comparison, all parameters of detected microorganisms and ARGs were calculated, including sequence number, relative abundance, genome coverage and depth, etc. 4) Report generation: The report generated by the system included all the microorganisms and ARGs in the specific microorganisms detected in the test. They classified by bacteria, viruses, fungi, parasites and ARGs, which were ranked from high to low according to their unique reads, and the relative content of the former was higher. In the report, ARG was shown as gene microorganism, such as adeB_A. baumannii. Only bacteria and ARGs were further analyzed in this study. Unique reads are number of sequences strictly mapped at species level or ARGs. 5) Threshold criteria for bacteria and ARGs as a positive result: (i) For bacteria except mycobacterium tuberculosis: bacteria species whose unique reads were of the first rank with the genus unique reads $>50$, cover length $>3000 \mathrm{bp}$. For mycobacterium tuberculosis: unique reads $\geq 3$. (ii) For ARGs: unique reads $>50$, cover length $>200 \mathrm{bp}$, cover percentage $>70 \%$. (iii) bacterium was reported as antibiotic resistance bacterium (ARB) in this study, if bacterium and ARG in this bacterium were positive simultaneously according the above criteria. Classification of ARGs resistance mechanism was carried out according to the CARD database.

\section{Statistical Analysis}

Prism (version 8.0, GraphPad Software, Inc, La Jolla, California, USA) and SPSS (version 22.0, IBM Corporation, Armonk, NY, USA) statistical software were used. Categorical variables described as n (\%) and Chi-square test or Fisher's exact test were used for categorical variables, as appropriate. Statistical significance was defined as $5 \%(\mathrm{P}<0.05)$.

\section{Results \\ Patient Characteristics}

In this study, a total of 504 clinical specimens from 452 patients were collected, 19 specimens $(3.77 \%, 19 / 504)$ of 18 patients were failed to get final results for variable reasons: small library fragments (often less than 100bp, considered as adapter dimer), lower library concentration/total reads, poor sequencing quality. The 19 failed specimens included 9 blood (9.27\%, 9/97), 6 BALF $(2.11 \%, 6 / 284), 2$ sputum $(2.06 \%, 2 /$ 97), 2 CSF (14.28\%, 2/14). 434 patients with successful mNGS results, included 312 males and 122 females. Mean age of all patients was $61.03( \pm 15.18)$ years, ranging from 0 to 96. Among them, 262 patients $(60.37 \%)$ had underlying different comorbidities, including cardiovascular disease (64, $14.71 \%)$, malignancies $(50,11.49 \%)$, respiratory disorders $(31,7.13 \%)$, renal/liver disease (23, 5.29\%), etc.; 377 patients $(86.67 \%)$ were treated with one or different combinations of antibiotic prior to sample collection (Table 1). 
Table I Demographic Characteristics of ICU Admission Patients with Pulmonary Infection

\begin{tabular}{|c|c|c|c|c|}
\hline & \multirow[t]{2}{*}{$\mathbf{N}(\%)$} & \multicolumn{2}{|c|}{ Bacteria with ARGs Detected } & \multirow[t]{2}{*}{$P$} \\
\hline & & Yes & No & \\
\hline \multicolumn{5}{|l|}{ Gender } \\
\hline Male & $312(7 \mid .89 \%)$ & 144 (46.15\%) & 168 (53.85\%) & \\
\hline Female & $122(28.11 \%)$ & $48(39.34 \%)$ & $74(60.66 \%)$ & 0.237 \\
\hline Age & $61.03 \pm 15.18$ & & & \\
\hline$<18$ & $22(5.07 \%)$ & 9 (40.91\%) & $13(59.09 \%)$ & \\
\hline $18-30$ & $15(3.46 \%)$ & $4(26.67 \%)$ & II (73.34\%) & \\
\hline $3 I-60$ & I4I (32.49\%) & $63(44.68 \%)$ & 78 (55.32\%) & \\
\hline $61-96$ & $256(58.99 \%)$ & II 6 (45.3I\%) & 140 (54.69\%) & 0.5504 \\
\hline \multicolumn{5}{|l|}{ Antibiotic history } \\
\hline Yes & $376(86.64 \%)$ & $167(44.41 \%)$ & 209 (55.59\%) & \\
\hline No & $58(13.36 \%)$ & $25(43.10 \%)$ & $33(56.90 \%)$ & 0.8878 \\
\hline \multicolumn{5}{|l|}{ Department } \\
\hline GICU & $306(70.51 \%)$ & I 48 (48.37\%) & 158 (5I.63\%) & \\
\hline $\mathrm{PICU}$ & $16(3.69 \%)$ & 7 (43.75\%) & $9(56.25 \%)$ & \\
\hline RICU & $89(20.51 \%)$ & $28(31.46 \%)$ & $61(68.54 \%)$ & \\
\hline SICU & $7(1.61 \%)$ & $5(71.43 \%)$ & $2(28.57 \%)$ & \\
\hline EICU & $16(3.69 \%)$ & $4(25.00 \%)$ & $12(75.00 \%)$ & 0.014 \\
\hline \multicolumn{5}{|l|}{ Comorbidities } \\
\hline None & 172 (39.63\%) & $72(4 \mid .86 \%)$ & $100(58.14 \%)$ & \\
\hline Respiratory disorders & $36(8.29 \%)$ & $13(36.12 \%)$ & $23(63.88 \%)$ & \\
\hline Malignancies & 51 (11.75\%) & $19(37.26 \%)$ & 32 (62.74\%) & \\
\hline Brain disease & 37 (8.53\%) & 24 (64.87\%) & $13(35.13 \%)$ & \\
\hline Cardiovascular disease & 37 (8.53\%) & 17 (45.95\%) & $20(54.05 \%)$ & \\
\hline Renal+ liver disease & 21 (4.84\%) & $8(38.10 \%)$ & $13(61.90 \%)$ & \\
\hline Diabetes/diabetes +others & $20(4.61 \%)$ & $10(50.0 \%)$ & $10(50.0 \%)$ & \\
\hline Autoimmune diseases & $18(4.15 \%)$ & 8 (44.45\%) & $10(55.55 \%)$ & \\
\hline Trauma & $8(1.84 \%)$ & $5(62.50 \%)$ & $3(37.50 \%)$ & \\
\hline Organ transplantation & II (2.53\%) & $8(72.73 \%)$ & $3(27.27 \%)$ & \\
\hline Others & $23(5.30 \%)$ & $8(34.79 \%)$ & $15(65.21 \%)$ & 0.1351 \\
\hline
\end{tabular}

\section{Frequency of Bacteria with ARGs in Clinical Samples}

A total of 329 bacteria with ARGs of 30 species were identified in 485 clinical samples with mNGS results from 434 patients, one or more bacteria with ARGs were detected in samples from 192 patients (44.24\%, 192/ 434). Among those, poly bacteria with ARGs were detected in samples from $85(19.59 \%, 85 / 434)$ patients. The bacteria with ARGs positive result was associated with the ICU department $(P<0.014)$ but gender, age, antibiotic history and comorbidities (Table 1). But All 30 bacteria species with ARGs were detected in the patients of male, age $\geq 61$, with antibiotic therapy history, GICU and with comorbidities (Figure 1A). The predominant detected bacteria with ARGs were
Corynebacterium striatum (C. striatum) (11.76\%, 51/ 434), A. baumannii $(11.52 \%, 50 / 434)$ and E. faecium (8.99\%, 39/434) (Figure 2). Two Mycobacterium tuberculosis ( $M$. tuberculosis), one with efpA and $m$ trA, another with efpA alone, and one Legionella pneumophila (L. pneumophila) with APH(9)-Ia were detected in BALF samples of three different patients. It was worth noting that one of the M. tuberculosis (efpA) was detected from BALF of a newborn girl, and A. baumannii (adeJ, adeF), E. faecium (ermB), Streptococcus pneumoniae (S. pneumoniae) (patB) were detected simultaneously.

The most prevalent bacteria with ARGs detected in male patients were C. striatum (41/254), A. baumannii (39/254) and E. faecium (34/254), while A. baumannii (13/75), C. striatum $(10 / 75)$ and $S$. pneumonia $(9 / 75)$ in female patients; In patients 


\section{A}

Corynebacterium striatum Acinetobacter baumannii Streptococcus pneumoniae Enterococcus faecium

Stenotrophomonas maltophilia Klebsiella pneumoniae

Staphylococcus epidermidis

Pseudomonas aeruginosa

Staphylococcus aureus Escherichia col

Staphylococcus haemolyticus

Elizabethkingia anophelis

Corynebacterium propinquum Enterococcus avium Burkholderia cepacia

Achromobacter xylosoxidans

Burkholderia multivorans

Staphylococcus hominis Corynebacterium aurimucosum

Mycobacterium tuberculosis

Corynebacterium kroppenstedtii Serratia marcescens Burkholderia stabilis Morganella morganii Nocardia farcinica

Streptococcus pyogenes

Legionella pneumophila

Pseudomonas geniculata Burkholderia cenocepacia Pseudomonas otitidis

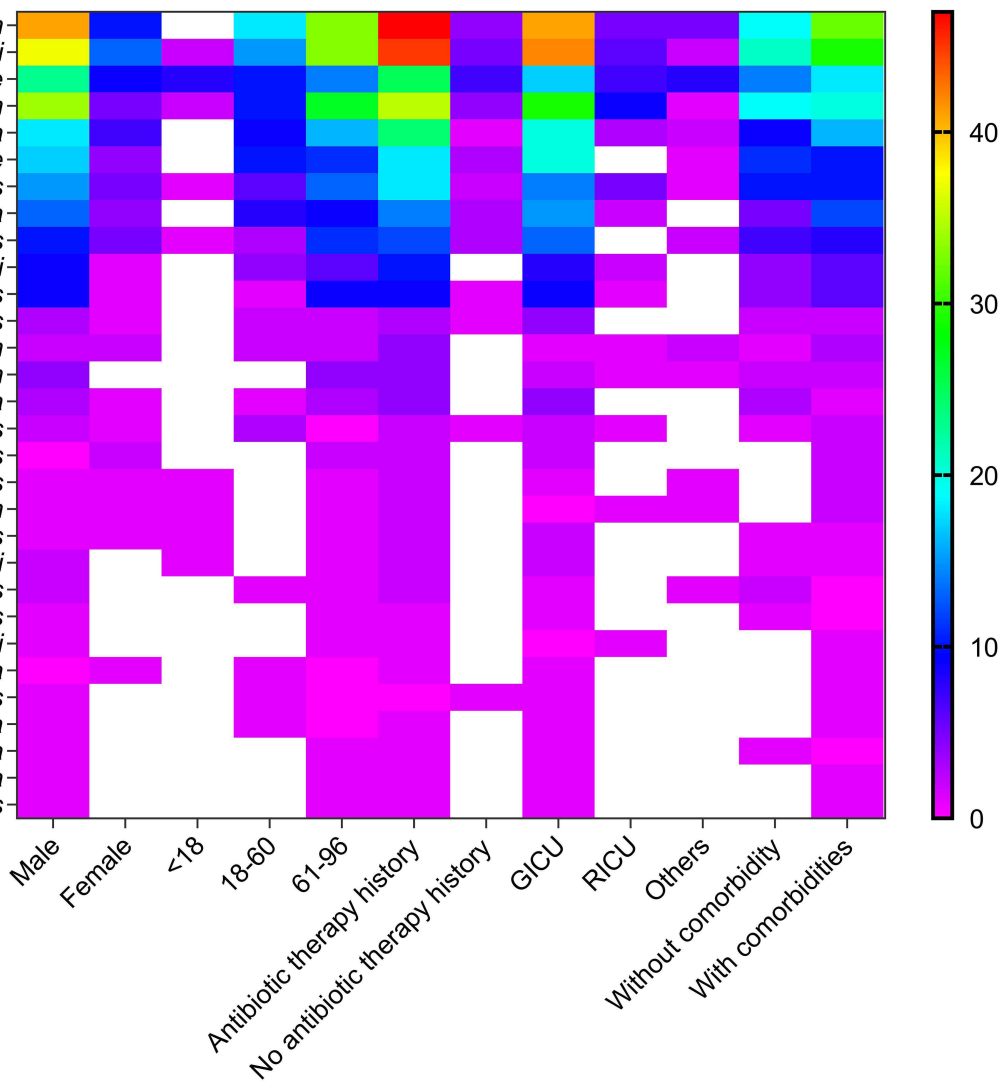

B

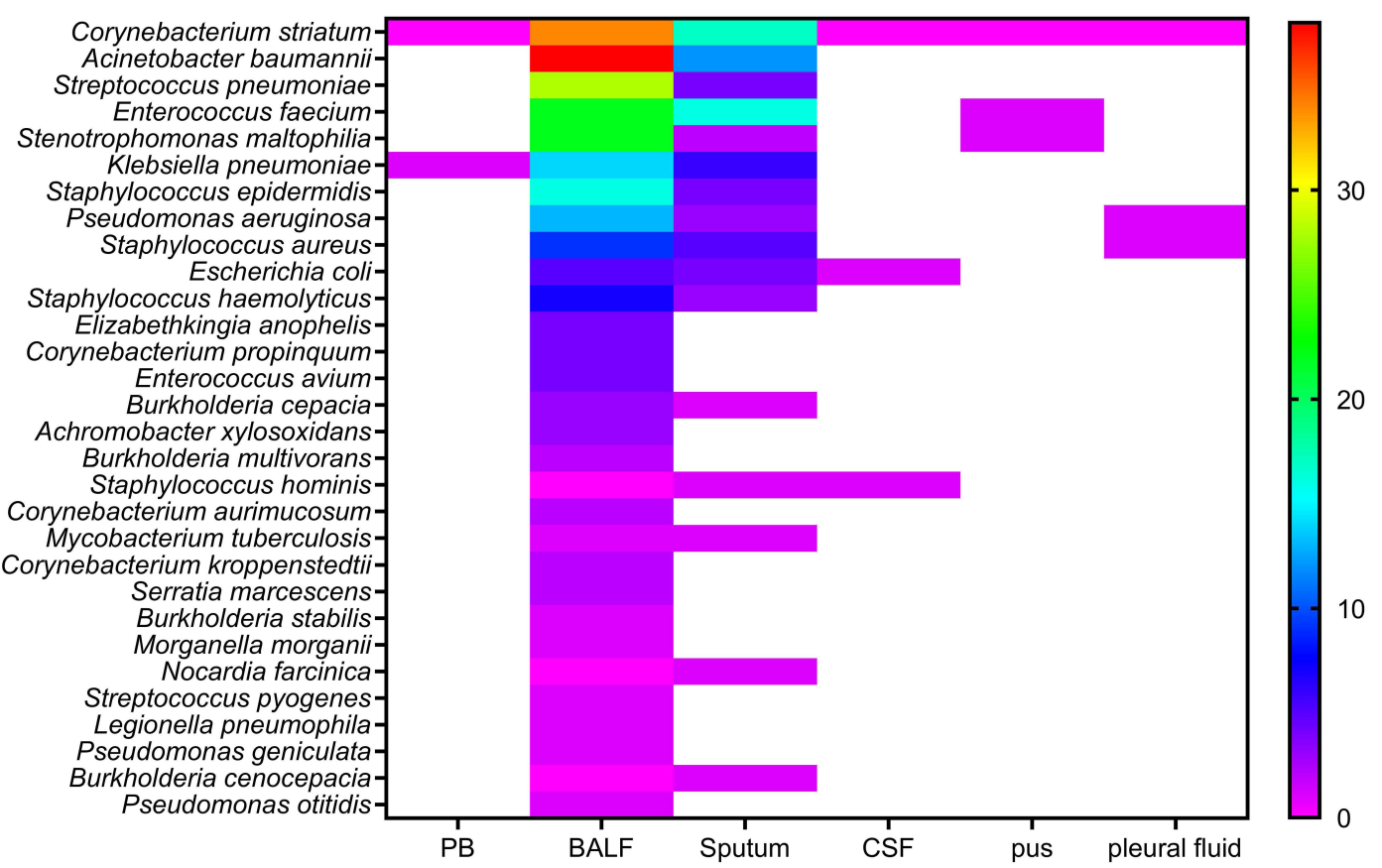

Figure I Spectrum and absolute number of bacteria with ARGs detected in samples from ICU admission patients with pulmonary infection. (A) Spectrum and absolute number of bacteria with ARGs detected in samples from different patients groups. (B) Spectrum and absolute number of bacteria with ARGs detected in different types of samples. 


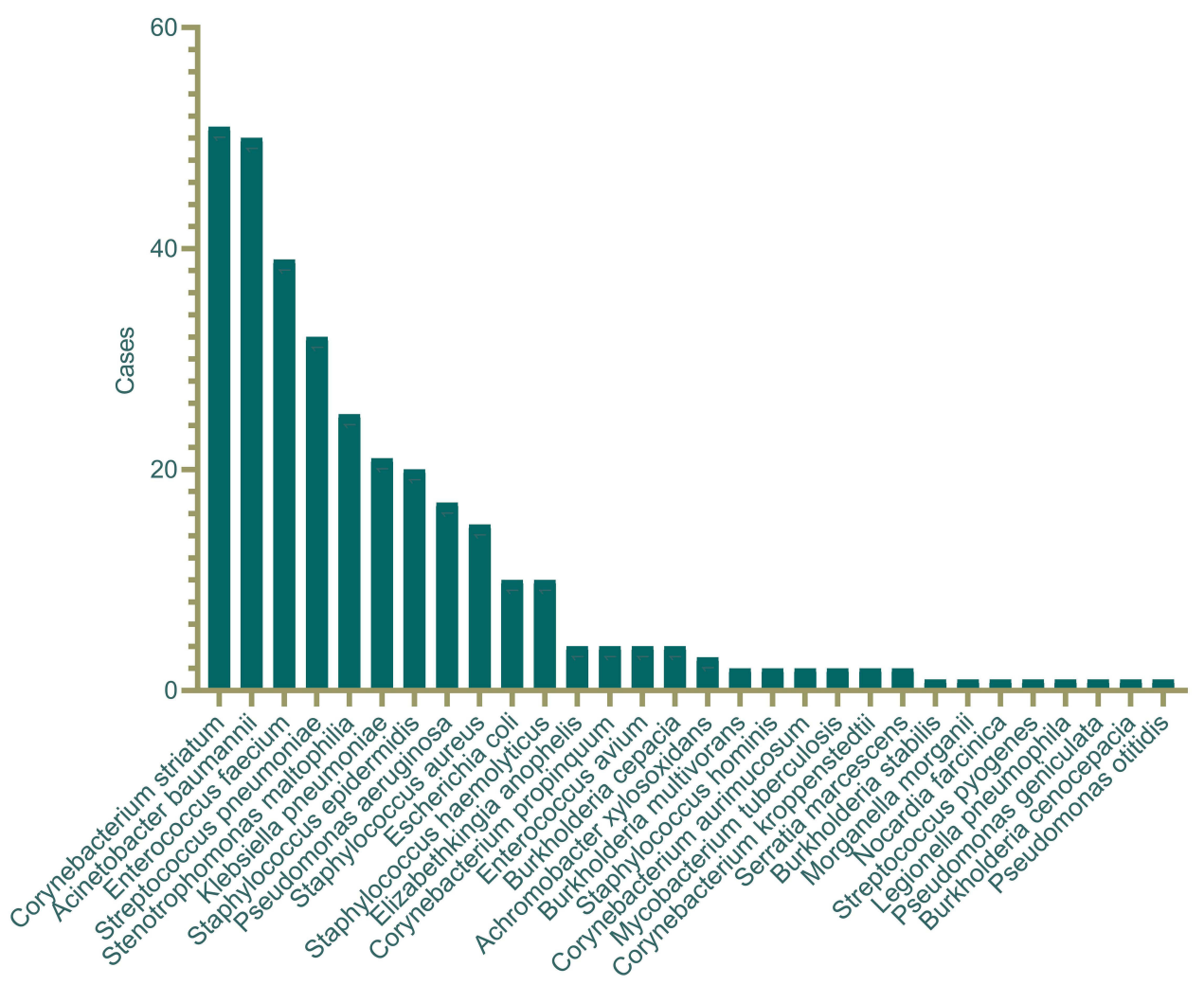

Figure 2 Long tail of bacteria with ARGs identified by mNGS in clinical samples from ICU admission patients with pulmonary infection.

with age $<18$, the most frequent detected bacteria with ARGs was $S$. pneumonia (8/18), while C. striatum $(18 / 106,33 / 205)$, A. baumannii $(15 / 106,33 / 205)$ in age $18-60$ and $\geq 61$, respectively. Among the patients with previous antibiotic histories, the predominant detected bacteria with ARGs were C. striatum (47/293), A. baumannii (45/293) and E. faecium (35/293) and $S$. pneumoniae (7/36) in patients without antibiotic history. In GICU, A. baumannii (42/256), C. striatum (41/256) and E. faecium (29/256) were the most prevalent bacteria with ARGs, while E. faecium (9/45) in RICU and $S$. pneumoniae (8/28) in others; C. striatum (19/138, 32/191), A. baumannii $(21 / 138,29 / 191)$ and E. faecium $(19 / 138,20 / 191)$ in patients with none and other comorbidities respectively (Figure 1A).

\section{Characteristics of Bacteria with ARGs in Different Types of Samples}

An average of $13.5 \mathrm{M}$ reads were generated for each sample of PB and CSF samples, similar with the sequencing data of BALF and sputum (average 14.3M for each sample). The difference in the sensitivity of bacteria with ARGs detected by mNGS between BALF $(50 \%, 140 / 280)$ and sputum $(50.5 \%, 48 / 95)$ was not statistically significant. However, the sensitivity was significantly higher for BALF, sputum samples than for PB, CSF samples (Figure 3 and Table S1). We did not analyze the differences in other types of sample for few cases, which would undermine the reliability of the results. In $88 \mathrm{~PB}$ samples with mNGS results, only one sample was detected $K$. pneumonia (OmpK37, acrA). Bacteria with ARGs were detected from two CSF samples, Escherichia coli (acrB, msbA, $m d t B$, evgS) in one sample, Staphylococcus haemolyticus (ermC) and C. striatum $(e r m X)$ in another. In total, 241 bacteria with ARGs were detected from 280 BLAF samples, and A. baumannii (38/ 241), C. striatum (34/241) and S. pneumoniae (28/241) were the most common bacteria with ARGs. While in 81 bacteria with ARGs detected from 95 sputum samples, the most frequency bacteria were C. striatum (17/81) and E. faecium (16/81) (Figure 1B).

In $31 \mathrm{BALF}+\mathrm{PB}$ paired samples, K. pneumonia (OmpK37, acr $A$ ) was detected in one BALF-PB paired sample simultaneously. There was no bacteria with ARGs detected simultaneously in paired sputum-PB samples. Bacteria with ARGs were observed simultaneously in one BALF-sputum paired sample, K pneumonia (OmpK37, acrA), A. baumannii (adeJ, adeB) were detected in BALF, while A. baumannii (adeJ, adeB), C. striatum (ermX, cmx), E. faecium (efmA) in sputum. 

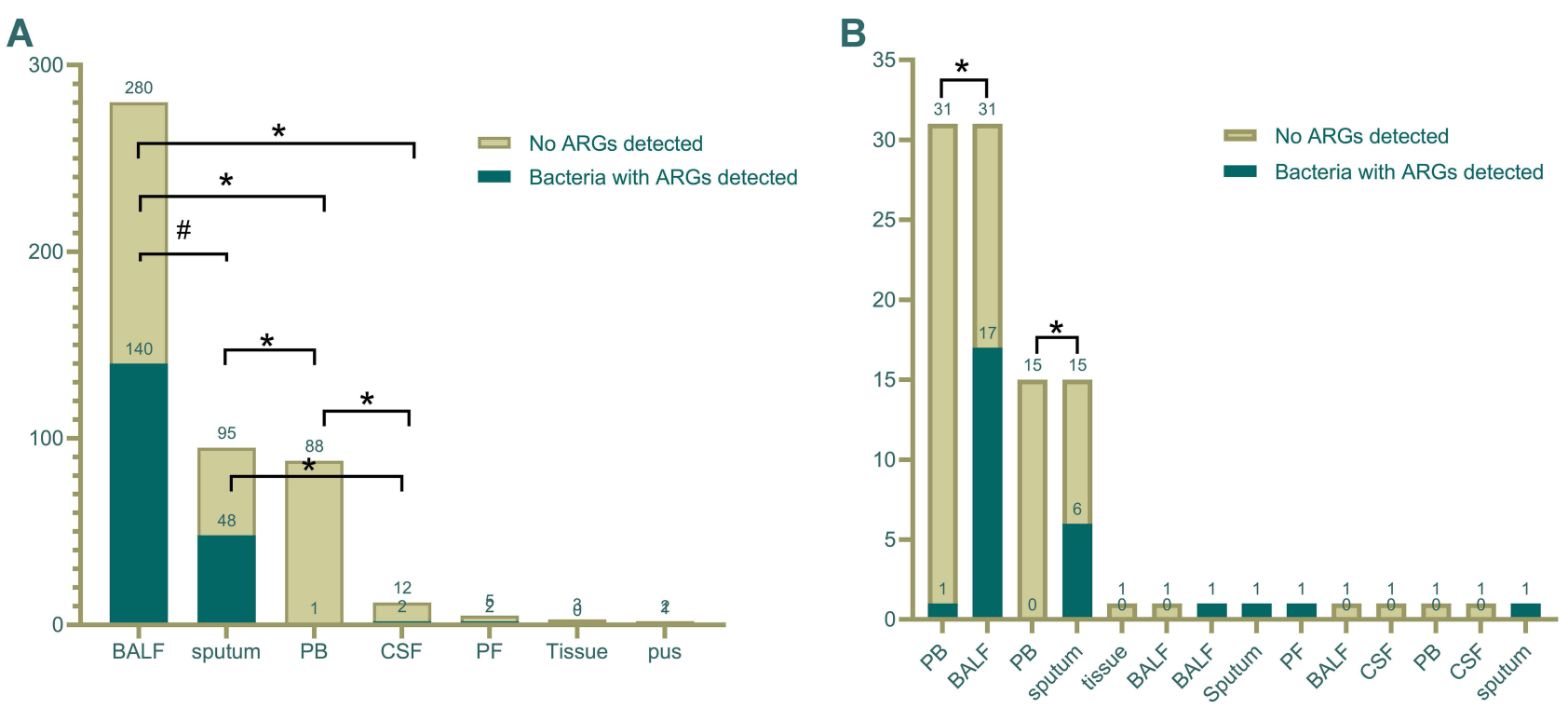

Figure 3 Comparison of bacteria with ARGs detected by $\mathrm{mNGS}$ from different types of samples. (A) Bacteria with ARGs detected in different types of samples. (B) Bacteria with ARGs detected in paired-sample from same patients. " Indicate no statistical significance; *Indicate statistical significance.

Abbreviation: PF, pleural fluid.

\section{Identification of Antibiotic Resistance Genes (ARGs)}

In total, 538 ARGs were detected in all 485 clinical specimens. By antibiotic class, these ARGs comprised multidrug resistance $(\mathrm{n}=220)$, Macrolide-LincosamideStreptogramin B $\left(\mathrm{MLS}_{\mathrm{B}}\right)(\mathrm{n}=123)$, tetracycline $(\mathrm{n}=$ 53), aminoglycoside $(n=42), \beta$-lactam $(n=39)$, phenicol $(\mathrm{n}=23)$, unclassified $(\mathrm{n}=17)$, fluoroquinolone $(\mathrm{n}=11)$ and others $(\mathrm{n}=10)$ genes (Table S2). Most of the multidrug resistance genes were efflux-associated genes. erm $X$ conferred resistance to $\mathrm{MLS}_{\mathrm{B}}$ and $\mathrm{cm} x$ to phenicol were the two resistance genes detected in C. striatum; In A. baumannii, the most frequently detected ARGs were adeJ and $a d e B$, which conferred resistance to multi-drug; while $\operatorname{erm} B$ conferred resistance to $\mathrm{MLS}_{\mathrm{B}}$ and efmA to multi-drug were the most prevalent ARGs detected in E. faecium. mel conferred resistance to multi-drug was most detected in S. pneumoniae, while smeB and smeE in Stenotrophomonas maltophilia; In $K$. pneumonia, ompK37 conferred resistance to $\beta$-lactam and acrA to multi-drug were the most common ARGs, while MexB and TriC in P. aeruginosa (Table S3).

\section{Discussion}

In this retrospective study, we have reported the mNGS analysis of 504 clinical specimens from 452 patients, and $3.77 \%(19 / 504)$ specimens were failed to get final mNGS result for variable reasons as described in results. Similar to other routine test in clinical laboratory, a wide range of pre-analytical variables may impact mNGS analysis, including specimen quality, storage and transit time, temperature, pre-analytical handling steps, etc. Studies showed that sputum quality affected the yield of M. tuberculosis diagnostic tests using smear and/or culture. ${ }^{66}$ The number of cells and red blood cells rarely interfered with $M$. tuberculosis detection by PCR in sputum samples, but a high concentration of cells and erythrocytes interfered with mycobacterial detection in BLAF and PF samples. ${ }^{67}$ There are no studies assessing the impact of specimen quality on mNGS assay till now. In this study, we did not perform sample quality evaluation before sample processing. Pre-analytical handling of clinical specimens is vital for the successful implementation of mNGS analysis. However, there is still no published standard operating procedure for sampling despite increasing studies on mNGS. A single-spin low-speed plasma separation, which is used in our study, is a feasible and inexpensive pre-analytical step for the recovery of pathogen DNA from blood. ${ }^{64}$ Although there was a study describing that freezing and thawing of plasma after storage at $-80^{\circ} \mathrm{C}$ for up to 6 months did not influence the abundance of pathogen, it should not undergo $>3$ freeze-thaw cycles. ${ }^{65}$ Induced sputum samples from patients need to be dispersed before analysis, and among several methods, the method utilizing DTT is widely used for its ability to split 
disulfide bonds and disperse sputum samples. ${ }^{68}$ We used the same method to proceed sputum samples before DNA extraction in our study.

Although it is still not a routine identification tool, mNGS presents great potential in resistance prediction by sequencing the ARGs. In our study, we explored the prevalence and distribution of bacteria with ARGs in clinical samples from real-world ICU admission patients with pulmonary infection in Mainland, China, using mNGS. In our study, bacteria with ARGs were detected in $44.24 \%$ patients and one or more bacteria with ARGs were detected in $19.54 \%$ cases. The predominant detected bacteria with ARGs were C. striatum, A. baumannii and, E. faecium. Of the top 11 most frequently bacteria with ARGs in our study, 5 (A. baumannii, Stenotrophomonas maltophilia, K. pneumonia, P. aeruginosa, Escherichia coli) were Gramnegative and 6 (C. striatum, E. faecium, S. pneumoniae, Staphylococcus epidermidis, S. aureus, Staphylococcus haemolyticus) were Gram-positive pathogens. But only one Gram-positive pathogen $S$. aureus, was among the top 11 most frequently isolated organism from the patients with ventilator-associated pneumonia in the United States and European medical centers from January 2009 to December 2012, and P. aeruginosa was the most frequently antibiotic resistance bacteria in both regions, followed by Klebsiella spp, Escherichia coli ranked fifth in the United States and third in Europe. ${ }^{10}$ In a study of 726 patients with nosocomial pneumonia, the most frequently isolated ceftazidime-nonsusceptible pathogens were Enterobacteriaceae, S. aureus and P. aeruginosa. ${ }^{11}$ The difference was caused by many factors, e.g. geographic areas, time, hospital unit, patients' specific characteristic, etc. According World Health Organization (WHO) global tuberculosis (TB) report (2020), China is still one of three highest drug-resistant TB burden countries worldwide. In our study, 2 drug-resistant M. tuberculosis were detected in samples from two different patients, and one was from BALF of a newborn girl.

C. striatum is non-sporing gram-positive bacterium, traditionally deemed to be a part of the normal flora of skin and mucous membranes, including the respiratory tract. It was first reported in 1980 as a pathogen ${ }^{12}$ and has been reported as an emerging multi-drug resistant nosocomial pathogen in recent studies. ${ }^{13-16}$ It has the potential to cause nosocomial infection in many parts of the body, and the respiratory tract is one of the main infection sites. ${ }^{17,18}$ Antibiotic-resistant C. striatum was detected in $11.73 \%$ patient samples and was the top one detected bacterium with ARGs in our study. High-level resistance to many antibiotics with different mechanisms such as the bla gene for penicillin resistance, ${ }^{13}$ gyrase subunit $A$ gene mutation for fluoroquinolones resistance $e^{21,24}$ and tet $A$, tet $B$ genes for tetracycline resistance ${ }^{25}$ in Corynebacterium have been reported. And high level resistance to macrolides, lincosamides, and streptogramin B $\left(\mathrm{MLS}_{\mathrm{B}}\right)$ in Corynebacterium is noticeable recently, especially in the multi-drug resistance clinical isolates. ${ }^{19,20}$ The ermX gene (erythromycin ribosome methylation) which may be found on plasmid, chromosomes and within transposon, encoding the rRNA methylase enzyme is responsible for $\mathrm{MLS}_{\mathrm{B}}$ resistance. ${ }^{21,22}$ The cmx gene encoding the efflux protein is responsible for phenicol resistance, and has been reported in several reports. ${ }^{14,21,23}$ In this study, only ermX and $c m x$ genes were found in the C. striatum, and erm $X$ was found in $98.04 \%(50 / 51)$ antibiotic resistant $C$. striatum.

ESKAPE represented $65.6 \%$ of all ventilator-associated pneumonia (VAP) isolates, and almost $20 \%$ being resistant. ${ }^{69}$ However, pulmonary infection due to Enterococcus, included E. faecium are distinctly unusual. A prospective and observational study showed that $4 \%$ infections located in the respiratory tract in patients with serious infection due to Enterococcus. ${ }^{70}$ Another study reported that 8 cases of pneumonia were due to $E$. faecium in 24 reported Enterococcus pneumonia, and 3 were antibiotic resistance. ${ }^{71}$ In our study, all the most serious ESKAPE with ARGs were detected in the clinical samples. A. baumannii in $11.50 \%$ patient samples, E. faecium in $8.97 \%$ patient samples, followed by $K$. pneumoniae, P. aeruginosa, S. aureus and Escherichia coli (Figure 2). A. baumannii is the most common clinical species responsible for ICU infection, accounting for high rates of various infections in ICU, especially pneumonia worldwide. ${ }^{26-28}$ A. baumannii is highly resistant to number of antibiotics in clinical practice because of its ability to acquire antimicrobial resistance. Efflux-mediated resistance, particularly the resistance-nodulation-division (RND)-type efflux pumps, is associated with many different classes of antibiotic resistance. In this study, All ARGs detected in A. baumannii were the RND-type efflux genes, including adeA, ade $B$, adeC, adeH, adeJ, adeK, adeF, adeL and adeI, except one with aminoglycoside resistance gene $A N T$ ( 3 ")-IIa. AdeJ and adeB were the most frequently ARGs among these genes, similar with the previous study in China. ${ }^{29}$ E. faecium is resistant to most clinical available antibiotics because of its intrinsic and acquired resistance mechanisms, including low-affinity PBPs to $\beta$-lactams, enzymatic inactivation to aminoglycosides, ribosomal methylation to aminoglycosides, ATP- 
binding cassette (ABC)-efflux pumps to $\mathrm{MLS}_{\mathrm{B}}$, precursor modification (VanA, $\operatorname{VanB})$ to glycopeptides, ribosomal methylation $(\mathrm{erm})$ to macrolides, ect. ${ }^{30,31}$ In our study, the most prevalent resistance determinants in E. faecium were erm $B$ conferring to $\mathrm{MLS}_{\mathrm{B}}$ and $e m f A$ conferring to macrolides and fluoroquinolone. Porins and efflux pumps are major contributors to antibiotic intrinsic resistance in gramnegative bacteria. ${ }^{32} \mathrm{OmpK} 35$, OmpK36 are two major porins in multidrug-resistant K. pneumonia. $^{32,33}$ In this study, OmpK37 was the most frequent porin gene in K. pneumonia. AcrAB of K. pneumonia, MexAB-OprM of $P$. aeruginosa, smeDEF and sme $A B C$ of $S$. maltophilia are the well-characterized efflux pumps with a demonstrated role in antibiotic resistance. ${ }^{34-39} A r c A, \operatorname{mexB}$ and $s m e B$ were the most prevalent efflux genes in $K$. pneumonia, $P$. aeruginosa and $S$. maltophilia respectively in our study. Estimated prevalence of methicillin-resistant $S$. aureus (MRSA) ranges in ICU admission patients is between $3 \%$ and $20 \%{ }^{43-46}$ The prevalence of MRSA in our study was $1.15 \%(5 / 435)$. The variation is more likely due to the geographic location and type of testing used. $S$. pneumoniae is the most common pathogen in community-acquired pneumonia (CAP), children with bacteremic pneumonia and ICU-admitted patients with pulmonary infection. ${ }^{40,41}$ Efflux-mediated resistance due to mefE and $m e l$ is a major mechanism of macrolide resistance in $S$. pneumoniae. ${ }^{42}$ In our study, the most common resistant determine in $S$. pneumoniae was mel (24/32), followed by $\operatorname{lnu} C(7 / 32)$.

For mNGS, the diagnostic sensitivity may vary in different types of samples. One study demonstrated that mNGS had higher sensitivity for detecting pathogen in BALF for pulmonary infection. ${ }^{47}$ But another study showed that there was no difference among pus, swab and tissue samples for skin and soft tissue infections. ${ }^{48}$ In our study, the bacteria with ARGs diagnostic sensitivity was higher in BALF, sputum than blood and CSF. Bacteria with ARGs were detected from 17/31 BALF specimens but only in $1 / 31$ corresponding blood specimens, while 6/ 15 from sputum specimens and none from corresponding specimens (Figure 3B). The prevalence of bacteria with ARGs is associated with the specimen types. Recent studies demonstrated that IMP-R $P$. aeruginosa were most frequently isolated from blood and sputum, MSRA from respiratory samples. ${ }^{49,50}$ In this study, the most common bacteria with ARGs in BALF were A. baumannii, C. striatum and S. pneumoniae, but C. striatum (17/81) and E. faecium (16/81) in sputum. And all the 30 species of bacteria with ARGs were detected from BALF specimens (Figure 1B). Considering sensitivity and spectrum of bacteria with ARGs detected, our results demonstrate that BALF, sputum specimens had advantages over $\mathrm{PB}, \mathrm{CSF}$ specimens for mNGS analysis used to detect the bacteria with ARGs in ICU patients with pulmonary infection.

Some limitations exist in this study. The application of mNGS remains controversial due to the limitations of mNGS technologies. The precision of mNGS is affected by many factors, such as the depth of mNGS, contamination of microbial nucleic acid in reaction kit, the microbehuman ratio in the original samples, the sample processing procedures, bioinformatics analysis and interpretation of sequencing result, etc. ${ }^{51,52}$ We used a validated mNGS-based approach according guideline ${ }^{58}$ and the ongoing monitoring, such as sample internal controls, swipe tests for contamination and periodic proficiency testing, etc., to minimize potential assay and patient selection bias, and it is an available assay for clinical reference testing of patients, but its clinical utility has not yet to be conducted in a large-scale prospective clinical trial. For small sample size, we still need more data to reflect the spectrum of antibiotic resistant determinants in the samples from ICU-admission patients with lung infection.

\section{Conclusion}

In this study, high level of bacteria with ARGs were observed in clinical samples, especially BALF, sputum samples from ICU admission patients with pulmonary infection. C. striatum with erm $X$ conferred resistance to $\mathrm{MSL}_{\mathrm{B}}$ and $c m x$ to phenicol, A. baumannii with adeJ and ade $B$ conferred resistance to multi-drug, and E. faecium with ermB to $M L S_{B}$, efmA to multi-drug were the most prevalent bacteria identified. The proportions of each bacteria with ARGs differed according the patients specific characteristic and sample types. BALF and sputum maybe were the optimal specimen type for mNGS to detect bacteria with ARGs for ICU patients with pulmonary infection. Identifying pathogen antibiotic resistance in ICU patients with pulmonary infection will support drug resistance monitoring, strengthen infection control in ICU in future research, and thus may contribute to improve antibiotic therapy.

\section{Data Sharing Statement}

All relevant data are provided in the manuscript, its figure, its table, its Supplementary Materials. 


\section{Ethical Approval and Informed Consent}

This study was approved by the Ethic Committee of CapitalBio Medlab. Written informed consent was obtained from each patient.

\section{Funding}

There is no funding to report.

\section{Disclosure}

The authors declare that there are no conflicts of interest in this work.

\section{References}

1. Mehta AB, Syeda SN, Wiener RS, et al. Epidemiological trends in invasive mechanical ventilation in the United States: a population-based study. J Crit Care. 2015;30(6):1217-1221. doi:10.1016/j.jcrc.2015.07.007

2. Luyt CE, Hékimian G, Koulenti D, et al. Microbial cause of ICU-acquired pneumonia: hospital-acquired pneumonia versus ventilator-associated pneumonia. Curr Opin Crit Care. 2018;24 (5):332-338. doi:10.1097/MCC.0000000000000526

3. Boucher HW, Talbot GH, Bradley JS, et al. Bad bugs, no drugs: no ESKAPE! An update from the infectious diseases society of America. Clin Infect Dis. 2009;48(1):1-12. doi:10.1086/595011

4. Moore LS, Freeman R, Gilchrist MJ, et al. Homogeneity of antimicrobial policy, yet heterogeneity of antimicrobial resistance: antimicrobial non-susceptibility among 108,717 clinical isolates from primary, secondary and tertiary care patients in London. J Antimicrob Chemother. 2014;69(12):3409-3422. doi:10.1093/jac/dku307

5. Kalil AC, Metersky ML, Klompas M, et al. Executive summary: management of adults with hospital-acquired and ventilator-associated pneumonia: 2016 clinical practice guidelines by the infectious diseases society of America and the American thoracic society. Clin Infect Dis. 2016;63(5):575-582. doi:10.1093/ cid/ciw504

6. Dananché C, Vanhems P, Machut A, et al. Trends of incidence and risk factors of ventilator-associated pneumonia in elderly patients admitted to French ICUs between 2007 and 2014. Crit Care Med. 2018;46(6):869-877. doi:10.1097/CCM.0000000000003019

7. Luna CM, Aruj P, Niederman MS, et al. Appropriateness and delay to initiate therapy in ventilator-associated pneumonia. Eur Respir J. 2006;27(1):158-164. doi:10.1183/09031936.06.00049105

8. MacVane SH. Antimicrobial resistance in the intensive care unit: a focus on gram-negative bacterial infections. $J$ Intensive Care Med. 2017;32(1):25-37. doi:10.1177/0885066615619895

9. Martins ST, Moreira M, Furtado GH, et al. Application of control measures for infections caused by multi-resistant gram-negative bacteria in intensive care unit patients. Mem Inst Oswaldo Cruz. 2004;99 (3):331-334. doi:10.1590/S0074-02762004000300017

10. Sader HS, Farrell DJ, Flamm RK, et al. Antimicrobial susceptibility of Gram-negative organisms isolated from patients hospitalised with pneumonia in US and European hospitals: results from the SENTRY Antimicrobial Surveillance Program, 2009-2012. Int J Antimicrob Agents. 2014;43(4):328-334. doi:10.1016/j.ijantimicag.2014.01.007

11. Torres A, Zhong N, Pachl J, et al. Ceftazidime-avibactam versus meropenem in nosocomial pneumonia, including ventilator-associated pneumonia (REPROVE): a randomised, double-blind, Phase 3 non-inferiority trial. Lancet Infect Dis. 2018;18(3):285-295. doi:10.1016/S1473-3099(17)30747-8
12. Bowstead TT, Santiago SM. Pleuropulmonary infection due to Corynebacterium striatum. Br J Dis Chest. 1980;74(2):198-200. doi:10.1016/0007-0971(80)90035-2

13. Alibi S, Ferjani A, Boukadida J, et al. Occurrence of Corynebacterium striatum as an emerging antibiotic-resistant nosocomial pathogen in a Tunisian hospital. Sci Rep. 2017;7(1):9704. doi:10.1038/s41598-017-10081-y

14. Wang X, Zhou H, Chen D, et al. Whole-genome sequencing reveals a prolonged and persistent intrahospital transmission of Corynebacterium striatum, an emerging multidrug-resistant pathogen. J Clin Microbiol. 2019;57(9):e00683-19. doi:10.1128/ JCM.00683-19

15. Shariff M, Aditi A, Beri K. Corynebacterium striatum: an emerging respiratory pathogen. J Infect Dev Ctries. 2018;12(7):581-586. doi: $10.3855 /$ jidc. 10406

16. McMullen AR, Anderson N, Wallace MA, et al. When good bugs go bad: epidemiology and antimicrobial resistance profiles of Corynebacterium striatum, an emerging multidrug-resistant, opportunistic pathogen. Antimicrob Agents Chemother. 2017;61(11):e0111117. doi:10.1128/AAC.01111-17

17. Verroken A, Bauraing C, Deplano A, et al. Epidemiological investigation of a nosocomial outbreak of multidrug-resistant Corynebacterium striatum at one Belgian university hospital. Clin Microbiol Infect. 2014;20(1):44-50. doi:10.1111/1469-0691.12197

18. Wang J, Wang Y, Du X, et al. Rapid transmission of multidrug-resistant Corynebacterium striatum among susceptible patients in a tertiary hospital in China. J Infect Dev Ctries. 2016;10 (12):1299-1305. doi:10.3855/jidc. 7577

19. Ortiz-Pérez A, Martín-de-hijas NZ, Esteban J, et al. High frequency of macrolide resistance mechanisms in clinical isolates of Corynebacterium species. Microb Drug Resist. 2010;16(4):273-277. doi: $10.1089 / \mathrm{mdr} .2010 .0032$

20. Otsuka Y, Ohkusu K, Kawamura Y, et al. Emergence of multidrug-resistant Corynebacterium striatum as a nosocomial pathogen in long-term hospitalized patients with underlying diseases. Diagn Microbiol Infect Dis. 2006;54(2):109-114. doi:10.1016/j. diagmicrobio.2005.08.005

21. Ramos JN, Souza C, Faria YV, et al. Bloodstream and catheter-related infections due to different clones of multidrug-resistant and biofilm producer Corynebacterium striatum. BMC Infect Dis. 2019;19(1):672. doi:10.1186/s12879-019-4294-7

22. Asgin N, Otlu B. Antimicrobial resistance and molecular epidemiology of Corynebacterium striatum isolated in a tertiary hospital in Turkey. Pathogens. 2020;9(2):136. doi:10.3390/pathogens9020136

23. Schröder J, Maus I, Meyer K, et al. Complete genome sequence, lifestyle, and multi-drug resistance of the human pathogen Corynebacterium resistens DSM 45100 isolated from blood samples of a leukemia patient. BMC Genomics. 2012;13:141. doi:10.1186/ 1471-2164-13-141

24. Eguchi H, Kuwahara T, Miyamoto T, et al. High-level fluoroquinolone resistance in ophthalmic clinical isolates belonging to the species Corynebacterium macginleyi. J Clin Microbiol. 2008;46 (2):527-532. doi:10.1128/JCM.01741-07

25. Campanile F, Carretto E, Barbarini D, et al. Clonal multidrug-resistant Corynebacterium striatum strains, Italy. Emerg Infect Dis. 2009;15(1):75-78. doi:10.3201/eid1501.080804

26. Inchai J, Pothirat C, Liwsrisakun C, et al. Ventilator-associated pneumonia: epidemiology and prognostic indicators of 30-day mortality. Jpn J Infect Dis. 2015;68(3):181-186. doi:10.7883/yoken.JJID.2014.282

27. Koulenti D, Tsigou E, Rello J. Nosocomial pneumonia in 27 ICUs in Europe: perspectives from the EU-VAP/CAP study. Eur $J$ Clin Microbiol Infect Dis. 2017;36(11):1999-2006. doi:10.1007/s10096016-2703-z

28. Vincent JL, Rello J, Marshall J, et al. International study of the prevalence and outcomes of infection in intensive care units. JAMA. 2009;302(21):2323-2329. doi:10.1001/jama.2009.1754 
29. Jia W, Li C, Zhang H, et al. Prevalence of genes of OXA-23 carbapenemase and AdeABC efflux pump associated with multidrug resistance of Acinetobacter baumannii isolates in the ICU of a comprehensive hospital of Northwestern China. Int $J$ Environ Res Public Health. 2015;12(8):10079-10092. doi:10.3390/ijerph120810079

30. Hollenbeck BL, Rice LB. Intrinsic and acquired resistance mechanisms in enterococcus. Virulence. 2012;3(5):421-433. doi:10.4161/ viru. 21282

31. Cattoir V, Giard JC. Antibiotic resistance in Enterococcus faecium clinical isolates. Expert Rev Anti Infect Ther. 2014;12(2):239-248. doi:10.1586/14787210.2014.870886

32. Fernández L, Hancock RE. Adaptive and mutational resistance: role of porins and efflux pumps in drug resistance. Clin Microbiol Rev 2012;25(4):661-681. doi:10.1128/CMR.00043-12

33. Palmeiro JK, de Souza RF, Schörner MA, et al. Molecular epidemiology of multidrug-resistant Klebsiella pneumonia isolates in a Brazilian tertiary hospital. Front Microbiol. 2019;10:1669. doi:10.3389/fmicb.2019.01669

34. Pan YP, Xu YH, Wang ZX, et al. Overexpression of MexAB-OprM efflux pump in carbapenem-resistant Pseudomonas aeruginosa. Arch Microbiol. 2016;198(6):565-571. doi:10.1007/s00203-016-1215-7

35. Horna G, López M, Guerra H, et al. Interplay between MexAB-OprM and MexEF-OprN in clinical isolates of Pseudomonas aeruginosa. Sci Rep. 2018;8(1):16463. doi:10.1038/s41598-018-34694-z

36. Mazzariol A, Zuliani J, Cornaglia G, et al. AcrAB efflux system: expression and contribution to fluoroquinolone resistance in Klebsiella spp. Antimicrob Agents Chemother. 2002;46 (12):3984-3986. doi:10.1128/AAC.46.12.3984-3986.2002

37. Bernardini A, Cuesta T, Tomás A, et al. The intrinsic resistome of Klebsiella pneumoniae. Int J Antimicrob Agents. 2019;53(1):29-33. doi:10.1016/j.ijantimicag.2018.09.012

38. Rizek CF, Jonas D, Garcia Paez JI, et al. Multidrug-resistant Stenotrophomonas maltophilia: description of new MLST profiles and resistance and virulence genes using whole-genome sequencing. J Glob Antimicrob Resist. 2018;15:212-214. doi:10.1016/j. jgar.2018.07.009

39. Chang LL, Chen HF, Chang CY, et al. Contribution of integrons, and SmeABC and SmeDEF efflux pumps to multidrug resistance in clinical isolates of Stenotrophomonas maltophilia. J Antimicrob Chemother. 2004;53(3):518-521. doi:10.1093/jac/dkh094

40. Fritz CQ, Edwards KM, Self WH, et al. Prevalence, risk factors, and outcomes of bacteremic pneumonia in children. Pediatrics. 2019;144 (1):e20183090. doi:10.1542/peds.2018-3090

41. Beumer MC, Koch RM, van Beuningen D, et al. Influenza virus and factors that are associated with ICU admission, pulmonary co-infections and ICU mortality. J Crit Care. 2019;50:59-65. doi:10.1016/j.jcrc.2018.11.013

42. Wierzbowski AK, Boyd D, Mulvey M, et al. Expression of the mef (E) gene encoding the macrolide efflux pump protein increases in Streptococcus pneumoniae with increasing resistance to macrolides. Antimicrob Agents Chemother. 2005;49(11):4635-4640. doi:10.1128/ AAC.49.11.4635-4640.2005

43. Izumikawa K, Yamamoto Y, Yanagihara K, et al. Active surveillance of methicillin-resistant Staphylococcus aureus with the BD GeneOhm MRSA $^{\text {TM }}$ assay in a respiratory ward in Nagasaki, Japan. Jpn J Infect Dis. 2012;65(1):33-36.

44. Veloso JO, Lamaro-Cardoso J, Neves LS, et al. Methicillin-resistant and vancomycin-intermediate Staphylococcus aureus colonizing patients and intensive care unit environment: virulence profile and genetic variability. APMIS. 2019;127(11):717-726. doi:10.1111/ apm. 12989

45. Ziakas PD, Anagnostou T, Mylonakis E. The prevalence and significance of methicillin-resistant Staphylococcus aureus colonization at admission in the general ICU setting: a meta-analysis of published studies. Crit Care Med. 2014;42(2):433-444. doi:10.1097/ CCM.0b013e3182a66bb8
46. Qiao F, Huang W, Cai L, et al. Methicillin-resistant Staphylococcus aureus nasal colonization and infection in an intensive care unit of a university hospital in China. J Int Med Res. 2018;46(9):3698-3708. doi:10.1177/0300060518777812

47. Wang Q, Wu B, Yang D, et al. Optimal specimen type for accurate diagnosis of infectious peripheral pulmonary lesions by mNGS. BMC Pulm Med. 2020;20(1):268. doi:10.1186/s12890-020-01298-1

48. Wang Q, Miao Q, Pan J, et al. The clinical value of metagenomic next-generation sequencing in the microbiological diagnosis of skin and soft tissue infections. Int J Infect Dis. 2020;100:414-420. doi:10.1016/j.ijid.2020.09.007

49. Hu YY, Cao JM, Yang Q, et al. Risk factors for carbapenem-resistant pseudomonas aeruginosa, Zhejiang Province, China. Emerg Infect Dis. 2019;25(10):1861-1867. doi:10.3201/eid2510.181699

50. Huang L, Zhang R, Hu Y, et al. Epidemiology and risk factors of methicillin-resistant Staphylococcus aureus and vancomycin-resistant enterococci infections in Zhejiang China from 2015 to 2017. Antimicrob Resist Infect Control. 2019;8:90. doi:10.1186/s13756019-0539-x

51. Simner PJ, Miller S, Carroll KC. Understanding the promises and hurdles of metagenomic next-generation sequencing as a diagnostic tool for infectious diseases. Clin Infect Dis. 2018;66(5):778-788. doi:10.1093/cid/cix881

52. Li H, Gao H, Meng H, et al. Detection of pulmonary infectious pathogens from lung biopsy tissues by metagenomic next-generation sequencing. Front Cell Infect Microbiol. 2018;8:205. doi:10.3389/fcimb.2018.00205

53. Yuan J, Li W, Qiu E, et al. Metagenomic NGS optimizes the use of antibiotics in appendicitis patients: bacterial culture is not suitable as the only guidance. Am J Transl Res. 2021;13(4):3010-3021.

54. Yee R, Breitwieser FP, Hao S, et al. Metagenomic next-generation sequencing of rectal swabs for the surveillance of antimicrobial-resistant organisms on the Illumina Miseq and Oxford MinION platforms. Eur J Clin Microbiol Infect Dis. 2021;40 (1):95-102. doi:10.1007/s10096-020-03996-4

55. Sukhum KV, Diorio-Toth L, Dantas G. Genomic and metagenomic approaches for predictive surveillance of emerging pathogens and antibiotic resistance. Clin Pharmacol Ther. 2019;106(3):512-524. doi:10.1002/cpt.1535

56. Langelier C, Kalantar KL, Moazed F, et al. Integrating host response and unbiased microbe detection for lower respiratory tract infection diagnosis in critically ill adults. Proc Natl Acad Sci U S A. 2018;115 (52):E12353-E12362. doi:10.1073/pnas.1809700115

57. Zinter MS, Dvorak CC, Mayday MY, et al. Pulmonary metagenomic sequencing suggests missed infections in immunocompromised children. Clin Infect Dis. 2019;68(11):1847-1855. doi:10.1093/cid/ ciy802

58. Schlaberg R, Chiu CY, Miller S, et al. Validation of metagenomic next-generation sequencing tests for universal pathogen detection. Arch Pathol Lab Med. 2017;141(6):776-786. doi:10.5858/ arpa.2016-0539-RA

59. Liapikou A, Cillóniz C, Torres A. Emerging strategies for the noninvasive diagnosis of nosocomial pneumonia. Expert Rev Anti Infect Ther. 2019;17(7):523-533. doi:10.1080/14787210.2019.1635010

60. Nicolau DP, Dimopoulos G, Welte T, et al. Can we improve clinical outcomes in patients with pneumonia treated with antibiotics in the intensive care unit? Expert Rev Respir Med. 2016;10(8):907-918. doi:10.1080/17476348.2016.1190277

61. Jeukens J, Freschi L, Kukavica-Ibrulj I, et al. Genomics of antibiotic-resistance prediction in Pseudomonas aeruginosa. Ann N Y Acad Sci. 2019;1435(1):5-17. doi:10.1111/nyas.13358

62. Angers-Loustau A, Petrillo M, Bengtsson-Palme J, et al. The challenges of designing a benchmark strategy for bioinformatics pipelines in the identification of antimicrobial resistance determinants using next generation sequencing technologies. F1000Res. 2018;7:459. doi:10.12688/f1000research.14509.2 
63. Chen X, Ding S, Lei C, et al. Blood and bronchoalveolar lavage fluid metagenomic next-generation sequencing in pneumonia. Can J Infect Dis Med Microbiol. 2020;2020:6839103. doi:10.1155/2020/6839103

64. Murugesan K, Hogan CA, Palmer Z, et al. Investigation of preanalytical variables impacting pathogen cell-free DNA in blood and urine. J Clin Microbiol. 2019;57(11):e00782-19. doi:10.1128/ JCM.00782-19

65. Han D, Li R, Shi J, et al. Liquid biopsy for infectious diseases: a focus on microbial cell-free DNA sequencing. Theranostics. 2020;10(12):5501-5513. doi:10.7150/thno.45554

66. Ho J, Marks GB, Fox GJ. The impact of sputum quality on tuberculosis diagnosis: a systematic review. Int J Tuberc Lung Dis. 2015;19 (5):537-544. doi:10.5588/ijtld.14.0798

67. Carnevale GG, Vargas FS, Caiaffa-Filho HH, et al. Preanalytical conditions can interfere with $\mathrm{M}$. tuberculosis detection by PCR in respiratory samples. Clinics. 2018;73:e410. doi:10.6061/clinics/2017/ e410
68. Hector A, Jonas F, Kappler M, et al. Novel method to process cystic fibrosis sputum for determination of oxidative state. Respiration. 2010;80(5):393-400. doi:10.1159/000271607

69. Sandiumenge A, Lisboa T, Gomez F, et al. Effect of antibiotic diversity on ventilator-associated pneumonia caused by ESKAPE organisms. Chest. 2011;140(3):643-651. doi:10.1378/chest.11-0462

70. Patterson JE, Sweeney AH, Simms M, et al. An analysis of 110 serious enterococcal infections. Epidemiology, antibiotic susceptibility, and outcome. Medicine. 1995;74(4):191-200. doi:10.1097/ 00005792-199507000-00003

71. Li F, Wang Y, Sun L, Wang X. Vancomycin-resistant Enterococcus faecium pneumonia in a uremic patient on hemodialysis: a case report and review of the literature. BMC Infect Dis. 2020;20(1):167. doi:10.1186/s12879-020-4892-4

\section{Publish your work in this journal}

Infection and Drug Resistance is an international, peer-reviewed openaccess journal that focuses on the optimal treatment of infection (bacterial, fungal and viral) and the development and institution of preventive strategies to minimize the development and spread of resistance. The journal is specifically concerned with the epidemiology of antibiotic resistance and the mechanisms of resistance development and diffusion in both hospitals and the community. The manuscript management system is completely online and includes a very quick and fair peerreview system, which is all easy to use. Visit http://www.dovepress.com/ testimonials.php to read real quotes from published authors. 